\title{
RSV prophylaxis guideline changes and outcomes in children with congenital heart disease
}

\author{
Adam S. Walpert MD ${ }^{1}$ (1) | Ian D. Thomas MD² | Merlin C. Lowe Jr MD MD $^{1}$ \\ Michael D. Seckeler MD, MSc ${ }^{3}$
}

${ }^{1}$ Division of Pediatric Hospital Medicine and Outreach, Department of Pediatrics, Banner Diamond Children's Medical Center,

University of Arizona, Tucson, Arizona, USA

${ }^{2}$ Division of Pediatric Cardiology, University of Michigan Congenital Heart Center, C.S Mott Children's Hospital, Ann Arbor, Michigan, USA

${ }^{3}$ Department of Pediatrics (Cardiology), University of Arizona, Tucson, Arizona, USA

\section{Correspondence}

Michael D. Seckeler, Department of Pediatrics (Cardiology), University of Arizona, 1501 N Campbell Ave, PO Box 245073, Tucson, AZ 85724, USA.

Email: mseckeler@peds.arizona.edu

\begin{abstract}
Objective: The aim of this study was to compare inpatient outcomes and costs for children with respiratory syncytial virus and congenital heart disease before and after the change in management guidelines for respiratory syncytial virus prophylaxis.

Design: Hospital discharge data from the Vizient (formerly University HealthSystem Consortium) were queried from October 2012 to June 2014 (Era 1) and July 2014 to April 2016 (Era 2) for patients aged $<24$ months with an any International Classification of Disease (ICD)-9 or ICD-10 code for congenital heart disease (745-747.49, Q20.0-Q26.4) and a primary or secondary admitting diagnosis of respiratory syncytial virus infection (079.6, J20.5), acute bronchiolitis due to respiratory syncytial virus (466.11, J21.0) or respiratory syncytial virus pneumonia (480.1, J12.1).
\end{abstract}

Setting: This study is a review of a national administrative discharge database.

Patients: Respiratory syncytial virus admissions were identified in 1269 patients aged $<24$ months with congenital heart disease, with 644 patients in Era 1 and 625 in Era 2. Patients 0-12 months old represented $83 \%$ of admissions.

Interventions: Prior to 2014, children aged 0-24 months with congenital heart disease were eligible to receive respiratory syncytial virus prophylaxis. Updated guidelines, published in 2014 , restricted the recommendation to administer palivizumab respiratory syncytial virus prophylaxis to children with congenital heart disease only if they are $\leq 12$ months old.

Outcome measures: The outcome measures are hospital length of stay, ICU admission rate, mortality, and direct costs.

Results: There was no change in length of stay, ICU admission rate, in-hospital mortality, or direct costs for children 13-24 months old with congenital heart disease after the change in guidelines. There were no deaths in 13-24 month olds, regardless of era.

Conclusions: Our findings provide additional support for the new guideline recommendations to provide respiratory syncytial virus prophylaxis only for children $\leq 12$ months old with congenital heart disease.

KEYWORDS

congenital heart disease, palivizumab, RSV

\section{1 | INTRODUCTION}

Respiratory syncytial virus (RSV) causes a lower respiratory tract infection in children leading to a high burden of disease. Morbidity and mortality are higher among premature infants, those with congenital heart disease (CHD), trisomy 21 and other diseases and malformations of the respiratory tract. ${ }^{1,2}$ Currently, there is no antimicrobial therapy with efficacy against RSV and treatment is primarily supportive. 
Palivizumab is a monoclonal antibody that can be given as a series of parenteral injections to provide passive immunity to high-risk children, thereby decreasing the severity of illness. In 2006, the American Academy of Pediatrics (AAP) released a clinical practice guideline recommending palivizumab prophylaxis against RSV for those children identified as high risk based on available data. ${ }^{3}$ Updated guidelines, published in July 2014, have decreased the number of patients with CHD who are recommended to receive palivizumab prophylaxis. Previously, children with hemodynamically significant cyanotic or acyanotic CHD were recommended to receive palivizumab if they were aged 0-24 months at the start of RSV season. This included children with congestive heart failure who required medical therapy and those with pulmonary hypertension. The 2014 guidelines restricted the recommended use of palivizumab to children with CHD only if they are $\leq 12$ months of age at the start of RSV season. ${ }^{4}$

The balance between the cost and benefit of palivizumab prophylaxis for children with CHD continues to be an area of debate on both a national and international level. As data have become available after the 2014 RSV prophylaxis guidelines revision, we are now able to better understand the effects. The goal of the present study is to describe the impact of the guideline changes on the outcomes and costs associated with RSV infection in hospitalized children $\leq 24$ months old with CHD.

\section{2 | METHODS}

Vizient (formerly University HealthSystem Consortium) is an alliance of 115 academic medical centers and 165 affiliated hospitals and their Clinical Data Base/Resource Manager (CDB/RM) is a large administrative database with discharge data from these institutions. ${ }^{5}$ After approval from the Institutional Review Board at the University of Arizona, we performed a retrospective review of de-identified discharge data from inpatient hospitalizations from the CDB/RM from October 2012 to April 2016. This study period allowed for two full RSV seasons before the guideline change (Era 1, 2012 and 2013) and two full RSV seasons after the guideline change (Era 2, 2014 and 2015). Of note, the International Classification of Disease, 10th edition (ICD-10) went into effect on October 1, 2015, so codes for ICD-9 and ICD-10 were used to query the database. Inclusion criteria included age $\leq 24$ months at admission with an any ICD-9 or ICD-10 code for CHD (745-747.49, Q20.0-Q26.4) and a primary or secondary admitting diagnosis of RSV infection (079.6, J20.5), acute bronchiolitis due to RSV (466.11, J21.0) or RSV pneumonia (480.1, J12.1). The data obtained in this query were for hospital admissions, and not individual patients, which makes tracking patients over time difficult. This data query only included inpatient admissions, as one of the primary benefits of RSV prophylaxis is to reduce RSV-associated hospitalizations.

Comparison variables included total length of stay (LOS), ICU admission rate, in-hospital mortality rate and direct costs. The data were compared between Era 1 and Era 2 for all admissions for 0-24 months old. Comparisons between Eras were made for 0-12 and 1324 months old to better understand the effect of changing the recommended age for palivizumab use from $\leq 24$ months to $\leq 12$ months old.
TABLE 1 Hospital outcomes and direct costs for all admissions for 0-24 month old patients with CHD and RSV, with comparisons between Eras (October 2012-June 2014 and July 2014-April 2016)

\begin{tabular}{|llll} 
& Era $1(n=644)$ & Era $2(n=625)$ & $P$ \\
\hline LOS (days) & $8.75 \pm 12.00$ & $9.49 \pm 16.17$ & .354 \\
\hline ICU admission rate (\%) & 40.18 & 47.2 & .013 \\
\hline Mortality, $n(\%)$ & $3(0.47)$ & $10(1.6)$ & .053 \\
\hline Direct costs (\$) & $13527 \pm 32437$ & $16055 \pm 39365$ & .224
\end{tabular}

Abbreviations: ICU, intensive care unit; LOS, length of stay. Data are presented as mean \pm standard deviation or $n(\%)$. Data from the Vizient Clinical Data Base/Resource Manager used by permission of Vizient. All rights reserved.

Data were analyzed using independent $t$-tests and chi-square or Fisher's exact test, as appropriate.

\section{3 | RESULTS}

A total of 1269 admissions for patients $\leq 24$ months old with CHD and RSV were identified for the entire study period, with 644 in Era 1 and 625 in Era 2. Comparisons between Eras for all admissions are shown in Table 1. Tables 2 and 3 show the comparisons for the $0-12$ and $13-24$ month groups, respectively. There were more admissions in the 0 12 month age group compared to the 13-24 month group (1057 vs 212).

Overall mortality was $1.02 \%$, with all of the mortality in the 0-12 month group and no deaths in the 13-24 month group in either Era. There was a nearly fourfold increase in hospital mortality for the 0-12 month group between Eras $(0.56 \%$ vs $1.93 \%, P=.051)$, which did not reach statistical significance. There was no change in mortality for the 13-24 month group. There was no difference in costs between the groups in either Era ( $\$ 14482$ vs $8187, P=.085$ and $\$ 17054$ vs $11109, P=0.171$ ), and there was no change in costs between Eras.

\section{DISCUSSION}

In this retrospective review of a large, national administrative database of children $\leq 24$ months old hospitalized with RSV infection, we found that there was no change in LOS, ICU admission rate, in-hospital

TABLE 2 Hospital outcomes and direct costs for all admissions for 0-12 month old patients with CHD and RSV, with comparisons between Eras (October 2012-June 2014 and July 2014-April 2016)

\begin{tabular}{llll} 
& Era $1(n=540)$ & Era $2(n=517)$ & $P$ \\
\hline LOS (days) & $9.27 \pm 12.58$ & $10.19 \pm 17.37$ & .323 \\
\hline ICU admission rate (\%) & 41.83 & 48.95 & .022 \\
\hline Mortality, $n(\%)$ & $3(0.56)$ & $10(1.93)$ & .051 \\
\hline Direct costs (\$) & $14482 \pm 34606$ & $17054 \pm 41657$ & .285
\end{tabular}

Abbreviations: ICU, intensive care unit; LOS, length of stay. Data are presented as mean \pm standard deviation or $n(\%)$. Data from the Vizient Clinical Data Base/Resource Manager used by permission of Vizient. All rights reserved. 
TABLE 3 Hospital outcomes and direct costs for all admissions for 13-24 month old patients with CHD and RSV, with comparisons between Eras (October 2012-June 2014 and July 2014-April 2016)

\begin{tabular}{lllr} 
& Era $1(n=104)$ & Era 2 $(n=108)$ & $P$ \\
\hline LOS (days) & $6.03 \pm 7.89$ & $6.12 \pm 7.53$ & .932 \\
\hline ICU admission rate (\%) & 31.65 & 38.89 & .316 \\
\hline Mortality, $n(\%)$ & $0(0)$ & $0(0)$ & 1.000 \\
\hline Direct costs (\$) & $8187 \pm 14468$ & $11109 \pm 24675$ & .322
\end{tabular}

Abbreviations: ICU, intensive care unit; LOS, length of stay. Data are presented as mean \pm standard deviation or $n$ (\%). Data from the Vizient Clinical Data Base/Resource Manager used by permission of Vizient. All rights reserved.

mortality or direct costs for children 13-24 months old with congenital heart disease after the change in RSV prophylaxis guidelines in July 2014. Using real-world data acquired after the change in RSV prophylaxis guidelines, these findings support the updated guidelines that no longer recommend RSV prophylaxis for children with CHD after 12 months of age.

Overall, there was little change in the measured outcomes among the study population, with the exception of an increased ICU admission rate and a trend towards an increase in hospital mortality from Era 1 to Era 2 in those aged 0-12 months, which is likely clinically important. Epidemiologic surveillance data have reported an increase of $12.75 \%$ in the incidence of RSV in between the two study Eras. ${ }^{6}$ This higher incidence may reflect a higher virulence of the RSV strains during Era 2, which could explain the increase in LOS and ICU admission and mortality rates identified, unrelated to the change in prophylaxis recommendations.

RSV prophylaxis generates potentially significant financial burdens on families and the healthcare system. A recent study estimated the cost of $\$ 4500$ for three doses of palivizumab, ${ }^{7}$ and extrapolating to a potential of six doses for an entire RSV season (November to April) could yield a cost as high as $\$ 9000$ per patient, assuming perfect compliance. By not providing prophylaxis to the 108 13-24 month olds with CHD in Era 2 of our study, there are significant potential cost savings with no change in clinical outcomes compared to Era 1.

A recent analysis of the CARESS Database in Canada found that children whose CHD remained hemodynamically significant beyond 12 months likely benefitted from continued RSV prophylaxis with fewer RSV hospitalizations. ${ }^{8}$ Our data query included all children with any diagnosis of $\mathrm{CHD}$, regardless of severity, so we were unable to assess for this potential effect. Chang has previously reported minimal change in hospitalization rates for patients with hemodynamically significant CHD after the 2003 RSV prophylaxis guidelines ${ }^{9}$, so we felt that looking at the entire CHD population would be reasonable for the current study.

There are limitations to the use of administrative databases for research purposes. Admissions were identified using ICD-9 and ICD-10 codes, which have limited detail for CHD, and may also be miscoded on entry into the database. However, previous reports have shown that ICD-9 codes in administrative databases have an $80 \%-85 \%$ specificity for congenital heart disease diagnoses. ${ }^{10}$ With the large numbers in our study, it is unlikely that enough admissions were miscoded in the database to have altered our findings. We were unable to isolate individual patient factors in this query, such as administration of palivizumab prior to hospital admission, multiple comorbidities in the same patient or specific medications which may have also increased hospital LOS, ICU admission rate and direct costs and could potentially limit the generalizability of our findings. While palivizumab administration rates were not assessed in our study, publicly available pharmaceutical data indicate a decrease in palivizumab revenue from \$1.04 billion $^{11}$ in 2012 to $\$ 662$ million $^{12}$ in 2015 . Despite these potential limitations, using a large administrative database allows us to look at a much larger population of patients than any one institution can provide to answer questions such as the implications of guideline changes.

\section{5 | CONCLUSION}

Using real-world data after changes in national guidelines for RSV prophylaxis for children $\leq 24$ months old with $\mathrm{CHD}$, we found no significant changes in the morbidity or mortality for hospitalized children with RSV and CHD between 13 and 24 months old. These findings support the new guideline recommendations to only provide RSV prophylaxis for children $\leq 12$ months old with $\mathrm{CHD}$. Longer term studies will be able to better define the impact on clinical outcomes and health care resource utilization.

\section{CONFLICT OF INTEREST}

The authors have indicated they have no potential conflicts of interest to disclose.

\section{FINANCIAL DISCLOSURE}

The authors have indicated they have no financial relationships relevant to this article to disclose.

\section{FUNDING SOURCE}

There are no funding sources for this study.

\section{AUTHOR CONTRIBUTIONS}

Drafted the initial manuscript, reviewed and revised the manuscript, and approved the final manuscript as submitted: Walpert

Conceptualized and designed the study, provided critical revision of the article, and approved the final manuscript as submitted: Thomas

Provided critical revision of the article, and approved the final manuscript as submitted: Lowe

Conceptualized and designed the study, carried out the initial analyses, reviewed and revised the manuscript, and approved the final manuscript as submitted: Seckeler

\section{ORCID}

Adam S. Walpert MD (iD http://orcid.org/0000-0003-4774-7534 Michael D. Seckeler MD, MSc (iD http://orcid.org/0000-0001-9013-5723 


\section{REFERENCES}

[1] Hall CB, Weinberg GA, Blumkin AK, et al. Respiratory syncytial virus-associated hospitalizations among children less than 24 months of age. Pediatrics. 2013;132(2):e341.

[2] Kristensen K, Hjuler T, Ravn H, Simoes EA, Stensballe L. Chronic diseases, chromosomal abnormalities, and congenital malformations as risk factors for respiratory syncytial virus hospitalization: a population-based cohort study. Clin Infect Dis. 2012;54(6): 810-817.

[3] Subcommittee on Management and Diagnosis of Bronchiolitis. Diagnosis and management of bronchiolitis. Pediatrics. 2006;118(4): 1774-1793.

[4] Ralston SL, Lieberthal AS, Meissner HC, et al. Clinical practice guideline: the diagnosis, management, and prevention of bronchiolitis. Pediatrics. 2014.

[5] Gutgesell HP, Hillman DG, McHugh KE, et al. Use of an administrative database to determine clinical management and outcomes in congenital heart disease. World J Pediatr Congenit Heart Surg. 2011; 2(4):593-596.

[6] Arizona Department of Health Services. Epidemiology \& Disease Control Flu \& RSV Reports-Home. http://www.azdhs.gov/preparedness/ epidemiology-disease-control/flu/index.php\#surveillance-home. Accessed May 27, 2016

[7] Blake SM, Tanaka D, Bendz LM, Staebler S, Brandon D. Evaluation of the financial and health burden of infants at risk for respiratory syncytial virus. Adv Neonatal Care. 2016;17:292-298.
[8] Li A, Wang DY, Lanctôt KL, Mitchell I, Paes BA. CARESS Investigators. Comparing first- and second-year palivizumab prophylaxis in patients with hemodynamically significant congenital heart disease in the CARESS database (2005-2015). Pediatr Infect Dis J. 2016;36: $445-450$.

[9] Chang RK, Chen AY. Impact of palivizumab on RSV hospitalizations for children with hemodynamically significant congenital heart disease. Pediatr Cardiol. 2010;31(1):90-95.

[10] Frohnert BK, Lussky RC, Alms MA, et al. Validity of hospital discharge data for identifying infants with cardiac defects. J Perinatol. 2005;25(11):737-742.

[11] Fourth Quarter and Full Year Results 2012. January 2013. https:// www.astrazeneca.com/content/dam/az/media-centre-docs/pressreleases/2013/fourth-quarter-full-year-results-2012/Press-release.pdf. Accessed October 12, 2017.

[12] Full-Year and Q4 2015 Results. February 2016. https://www.astrazeneca.com/content/dam/az/PDF/Fourth_quarter_and_Full_Year_results_ 2015_press_release.pdf. Accessed October 12, 2017.

How to cite this article: Walpert AS, Thomas ID, Lowe MC Jr, Seckeler MD. RSV prophylaxis guideline changes and outcomes in children with congenital heart disease. Congenital Heart Disease. 2018;13:428-431. https://doi.org/10.1111/chd.12590 